\title{
Designing and Evaluating Text Entry Methods
}

\author{
Per Ola Kristensson \\ University of St Andrews \\ St Andrews, UK \\ pok@st-andrews.ac.uk \\ James Clawson \\ Georgia Institute of Technology \\ Atlanta, Georgia, USA \\ jamer@cc.gatech.edu \\ Mark Dunlop \\ University of Strathclyde \\ Glasgow, UK \\ mark.dunlop@cis.strath.ac.uk \\ Poika Isokoski \\ University of Tampere \\ Tampere, Finland \\ poika.isokoski@uta.fi \\ Brian Roark \\ Oregon Health \& Science \\ University \\ Portland, Oregon, USA \\ roark@cslu.ogi.edu \\ Keith Vertanen \\ Montana Tech of the University of \\ Montana \\ Butte, Montana, USA \\ kvertanen@mtech.edu

\section{Annalu Waller} \\ University of Dundee \\ Dundee, UK \\ annalu.waller@dundee.ac.uk

\section{Jacob O. Wobbrock} \\ University of Washington \\ Seattle, Washington, USA \\ wobbrock@uw.edu \\ Copyright is held by the author/owner(s) \\ CHI'12, May 5-10, 2012, Austin, Texas, USA. \\ ACM 978-1-4503-1016-1/12/05.
}

\begin{abstract}
Our workshop has three primary goals. The first goal is community building: we want to get text entry researchers that are active in different communities into one place. Our second goal is to promote $\mathrm{CHI}$ as a natural and compelling focal point for all kinds of text entry research. The third goal is to discuss some difficult issues that are hard or near impossible to handle within the traditional format of research papers.

\section{Keywords}

Text entry; augmentative and alternative communication; accessibility; internationalization

\section{ACM Classification Keywords}

H.5.2 [Information interfaces and presentation]: User Interfaces-Input devices and strategies;

\section{General Terms}

Design, Experimentation, Human Factors

\section{Introduction}

Text entry is a culture preserving device [3] and therefore of tremendous importance for our society. As a consequence, it is not surprising that the art of designing new text entry methods has been practiced for hundreds of years. For example, monks in $12^{\text {th }}$
\end{abstract}


century Europe already used some of the techniques we use today when crafting text entry methods, such as word frequency analysis [3]. The technological developments during the $20^{\text {th }}$ and $21^{\text {st }}$ centuries have accelerated the need for better text entry methods for a wide array of application domains and devices. With email, instant messaging and social networking technologies, text entry since the late $20^{\text {th }}$ century has become the principle basis for communication. For users with motor impairments, or other disabilities, new text entry methods enable faster communication, which can dramatically improve people's quality of life.

Areas of active research include designing text entry methods for mobile phones (see $[3,5,16]$ for extensive overviews), video games (e.g. [14]), wall-sized displays (e.g. [8]), surfaces (e.g. $[1,2])$, wearable computers (e.g. [4]), and for augmentative and alternative communication (AAC) devices that help users with communication difficulties interact with the people around them (e.g. $[12,13,15])$. Another active research area is support for non-Western languages (e.g. [7]). However, these research efforts are today scattered across multiple communities, such as humancomputer interaction ( $\mathrm{HCI})$, augmentative and alternative communication (AAC), speech recognition, human factors, and accessible computing. There is a need to reach out and (re)connect with these communities outside $\mathrm{HCI}$ so that we can inspire and learn from each other. We strongly believe $\mathrm{CHI}$ has the potential to become the central nave for text entry research. However, for this to happen we have to actively arrange events which bring together people from all these communities.
There are also many research concerns that involve the entire text entry community. Some of these are about the design of our experiments and the metrics we choose to use. Other goals are about our legacy. Since text entry researchers are spread across different fields, many text entry methods are forgotten or described in insufficient detail for later generations of researchers to fully appreciate them. We therefore want to unify the text entry community and discuss how we can create a curated archive of text entry methods.

\section{Workshop goals}

- Community building: Today text entry researchers are scattered across the human-computer interaction, intelligent interactive systems,

experimental psychology, human factors, augmentative and alternative communication, natural language processing, and speech and signal processing communities. Furthermore, research in novel text entry solutions takes place in both academic and industrial research labs. We want to raise awareness of the research activities and priorities that concern people in different research fields and learn from each other's successes and failures.

- CHI focus: We want to make the SIGCHI community known to text entry researchers who may be more active in other communities (such as the augmentative and alternative communication or natural language processing fields). A goal of this workshop is to advertise $\mathrm{CHI}$ as a natural and compelling point of focus for all kinds of text entry research.

- A constructive research dialogue: Since researchers are scattered across different research fields, the scientific dialogue is equally scattered. People in different communities may not be aware of 
research progress and resolved controversies in neighboring fields. A major goal of this workshop is to bring all these people together to discuss some difficult issues that are hard or near impossible to handle within the traditional format of research papers.

\section{Panel topics for text entry}

The workshop will be structured as a series of panels that involve a large part of the text entry community. While we encourage suggestions for panel topics in our call for workshop papers, we believe the six panel topics below are of tremendous importance for our community:

- Ensure scientific quality of the highest caliber: Do we need to develop standards for text entry evaluations? Many metrics for evaluation have been proposed for measuring text entry performance within tightly controlled experiments (e.g. [9]). Similarly, there are attempts to standardize phrase sets (e.g. $[6,10])$. However, in the text entry literature, people often use incompatible methods for measuring text entry performance. Should we try to enforce a standard, and if so, how would such a standard be communicated and enforced?

\section{- Outreach and community building: How do we} reach out to the text entry research communities outside HCI? For example, how would we reengage with the AAC community? Currently there are concurrent efforts to design accessible text entry methods, such as text entry methods that minimize the amount of physical effort required to write text (e.g. [13]). By combining efforts we may increase the rate of progress in text entry research.
- Maintaining our legacy: How can we create and maintain a curated text entry archive for the benefit of current and future generations of text entry researchers? Many text entry methods risk becoming forgotten or ignored when new generations of researchers continue to create new methods. This is particularly so due to the scattered nature of text entry methods. For example, an $\mathrm{HCI}$ researcher may not realize that relevant text entry research may exist in the AAC community, and vice versa. We think a shared and clearly advertised high-quality archive of text entry methods developed and maintained by the active research community would be tremendously helpful for text entry researchers in all research fields.

- New environments and technologies: Which contexts, situations, and environments require, or will require, better text entry methods in the future? For example, as wall-sized displays and large surfaces become more prominent we predict that the demand for high-quality text entry methods will increase. Another underexplored topic in text entry is context of use-how do people actually use advanced text entry methods in, for instance, truly mobile tasks, such as writing while walking in a noisy airport terminal? How can we reliably measure text entry performance in such environments?

- Enabling technologies and methods: What new technologies, methods and techniques can generate new text entry methods? For instance, advances in signal processing, machine learning and sensor technologies may open up a range of new possible text entry methods. As an example, a recent paper used crowdsourcing and state-of-the-art natural language processing algorithms to create high-quality statistical language models for AAC devices [11]. 
- New metrics and goals: Are speed and accuracy always the best metrics to report in our publications? How do we measure the success of a new input system designed to improve literacy, or one that enables an individual to communicate with others for the first time ever? How should the goals of text entry research evolve and what metrics should we adopt as a community to ensure that we can meet these goals?

\section{Conclusions}

The text entry community is currently very active and regularly makes significant contributions to both the research literature and our society at large. However, our community is scattered across different research fields, such as HCI, AAC, speech recognition and natural language processing. There are also a number of topics that are difficult to discuss in the traditional format of technical papers. This workshop serves to unify the text entry community and center it at $\mathrm{CHI}$.

\section{References}

[1] Findlater, L., Wobbrock, J.O. and Wigdor, D. Typing on flat glass: examining ten-finger expert typing patterns on touch surfaces. In Proc. CHI 2011, ACM Press (2011), 2453-2462.

[2] Hinrichs, U., Hancock, M., Collins, C. and Carpendale, S. Examination of text-entry methods for tabletop displays. In Proc. Tabletop 2007, IEEE Press (2007), 105-112.

[3] Kristensson, P.O. Five challenges for intelligent text entry methods. AI Magazine 30, 4 (2009), 85-94.

[4] Lyons, K., Starner, T., Plaisted, D., Fusia, J., Lyons, A., Drew, A. and Looney, E.W. Twiddler typing: onehanded chording text entry for mobile phones. In Proc. CHI 2004, ACM Press (2004), 671-678.

[5] MacKenzie, I. S., and Soukoreff, R.W. Text entry for mobile computing: models and methods, theory and practice. Human-Computer Interaction 17, 2 (2002), 147-198.

[6] MacKenzie, I.S. and Soukoreff, R.W. Phrase sets for evaluating text entry technique. In Ext. Abstracts CHI 2003, ACM Press (2003), 754-755.

[7] Masui, T. 1998. An efficient text input method for pen-based computers. In Proc. CHI 1998, ACM Press (1998), 328-335.

[8] Olsen, D.R. and Nielsen, S.T. Laser pointer interaction. In Proc. CHI 2001, ACM Press (2001), 1722.

[9] Soukoreff, R.W. and MacKenzie, I.S. Metrics for text entry research: an evaluation of MSD and KSPC, and a new unified error metric. In Proc. CHI 2003, ACM Press (2003), 113-120.

[10] Vertanen, K. and Kristensson, P.O. A versatile dataset for text entry evaluations based on genuine mobile emails. In Proc. MobileHCI 2011, ACM Press (2011), 295-298.

[11] Vertanen, K. and Kristensson, P.O. The imagination of crowds: conversational AAC language modeling using crowdsourcing and large data sources. In Proc. EMNLP 2011, ACL (2011), 700-711.

[12] Waller, A. and Jack, K. A predictive Blissymbolic to English translation system. In Proc. ASSETS 2002, ACM Press (2002), 186-191.

[13] Ward, D. J., and MacKay, D. J. C. Fast hands-free writing by gaze direction. Nature 418 (2002), 838.

[14] Wilson, A.D. and Agrawala, M. Text entry using a dual joystick game controller. In Proc. CHI 2006, ACM Press (2006), 475-478.

[15] Wobbrock, J.O. and Myers, B.A. Trackball text entry for people with motor impairments. In Proc. $\mathrm{CHI}$ 2006, ACM Press (2006), 479-488.

[16] Zhai, S., Kristensson, P.O. and Smith, B.A. In search of effective text input interfaces for off the desktop computing. Interacting with Computers 17, 3 (2005), 229-250. 